\title{
Die Güte der Beziehung
}

\section{Wie relationale Güter sozial zustande kommen}

Wolf Rainer Wendt

Prof. Dr. Wolf Rainer Wendt ist

Vorsitzender der Deutschen Gesellschaft für Care und Case Management sowie Honorarprofessor der Eberhard Karls Universität Tübingen. Er ist Mitglied im Beirat der Blätter der Wohlfahrtspflege. E-Mailwendt@imails.de
Die von italienischen Ökonomen entwickelte " Theorie der relationalen Güter « gehört in die Diskussion von Qualitäten, die Familien und anderen Lebensgemeinschaften eigen sind und die in der Beziehungsgestaltung und zur Behebung von Beziehungsarmut durch Soziale Arbeit erzeugt werden. Professionell lässt sich Raum schaffen und Gelegenheit bieten für relationale Güter, die in der Partizipation von Personen aneinander zustande kommen.

Beziehungen haben ist gut, aber soziale Beziehungen sind in ihrer Art sehr verschieden und auch unterschiedlich zu bewerten. Es gibt Familienbeziehungen, Freundschaften, nachbarschaftliche Beziehungen, Liebesbeziehungen, kollegiale Beziehungen, Geschäftsbeziehungen. Sie sind nicht von gleicher Natur und Bedeutung, und unter sozialen Gesichtspunkten ist es angebracht, den Nutzen und den Wert verschiedener Beziehungen $\mathrm{zu}$ untersuchen, auch um Menschen zu helfen, denen es an ihnen mangelt. Viele Beziehungen lassen sich instrumentell für bestimmte Zwecke einsetzen (und bilden ein soziales Kapital); andere entziehen sich jedem Kalkül und werden um ihrer selbst willen gesucht. Im Folgenden sei die Klasse von Beziehungen näher betrachtet, die nicht von einer Person alleine unterhalten werden können und deshalb in einem besonderen Sinne sozialer Natur sind.

In der Wirtschaftswissenschaft wird klassisch von Individuen ausgegangen, die eigennützig handeln und in ihrem Handeln für sich einen Gewinn realisieren wollen. Indes hat bereits Adam Smith in seiner »Theorie der moralischen Gefühle " vom "fellow feeling " gesprochen und damit eine Sympathie gemeint, die auf geteilter Erfahrung basiert. In den letzten zwei Jahrzehnten haben nun einige, vor allem italienische, Ökonomen dasjenige »Gut « zum Gegenstand ihrer Erörterung gemacht, das nur im Mitein- ander von Menschen, durch ihr Involviertsein in Gemeinschaft existiert und in der Qualität ihrer Beziehungen gegeben ist (Uhlaner 1989, Gui 1996). Eine Person kann dieses »Beziehungsgut « (»relational good «) nicht für sich allein besitzen. Es ist nicht handelbar; seine Voraussetzung ist die Begegnung von Menschen und dass sie aneinander teilhaben. »One can define a relational good as one that produces utility to the consumer only if shared with other subjects. (Zamagni 1999, 225) Die Begegnung wird als ein produktiver Prozess verstanden, dem die Beteiligten für sich Gutes abgewinnen.

\section{Güter menschlicher Nähe}

Die Theorie relationaler Güter begreift »Begegnung « (»encounter «) als generierenden Vorgang der Erzeugung von Beziehungsgütern (Gui 2005). Sie sind sozial produzierte Güter, deren Besonderheit aus wirtschaftswissenschaftlicher Sicht darin besteht, dass weder der Markt noch der Staat sie zur Verfügung stellen kann, sondern nur die unmittelbare soziale Interaktion. Sie lassen sich nicht wie gewöhnliche Waren herstellen und liefern, sondern sind nur in der Gestalt menschlicher Beziehungen vorhanden ( $\mathrm{Sacco} / \mathrm{Va}-$ nin/Zamagni 2006, 699). Menschen nehmen sie wahr und pflegen sie. In einem relationalen Gut steckt persönliche Geltung und Anerkennung. Anonymität steht ihm entgegen; denn Güter » which arise in exchanges where anyone could anonymously supply one or both sides of the bargain are not relational « (Uhlaner 1989, 255).

Es geht um menschliche Nähe; sie kann aber nicht nur ein Gewinn, sondern auch eine Bedrängnis sein, wie wir sie in der Soziale Arbeit als »fürsorgliche Belagerung « kennen. Eine Beziehung als solche ist nicht schon das relationale Gut; erst ihr erfahrener Charakter beinhaltet die Gratifikation, die das relationale Gut für 
die an der Beziehung Beteiligten bedeutet. Wer sich im Besitz eines solchen Gutes weiß, nutzt es nicht aus. Wir haben von den hier gemeinten Beziehungsgütern die Statusgüter (»positional goods «) zu unterscheiden, die man in einer Machtposition besitzen oder als Sozialprestige genießen kann (Frank 1985). Bei Statusgütern ist eine (behauptete oder tatsächliche) Ungleichheit vorausgesetzt, und sie steht einer wirklichen Partizipation entgegen. Abgehoben sei von relationalen Gütern auch ein »Beziehungskapital « im Sinne eines Sozialkapitals, das sich nach dem Aufbau von Beziehungen instrumentell für andere Zwecke nutzen lässt. Ein relationales Gut kann weder Mittel zum Zweck sein noch zu einer Ware werden.

In der Erwerbsökonomie lassen sich die interpersonalen Qualitäten durchaus in den Austausch unter Handelspartnern einbeziehen - man schmiert damit das Geschäft - und in einem Produktionsbetrieb spielen gute Beziehungen unter den Mitarbeitern und das emotionale Klima in der Firma eine wichtige Rolle im »Throughput « der Leistungserstellung. Aber sie sind nicht erstrangig für den ökonomischen Prozess dort. In der Wohlfahrtspflege können relationale Güter nun aber das eigentliche Produkt sein, auf das in der Arbeit abgesehen und das in ihr generiert wird.

Menschen suchen von sich aus nach dem Gut der Begegnung und eines gepflegten Miteinanders. Man trifft sich, tauscht sich aus, kommuniziert miteinander - und das trägt zum Wohlbefinden bei. Individuell handeln Menschen so und begeben sich dazu in eine geeignet erscheinende Situation, wenn sie einen Bedarf an sozialer Teilhabe spüren. Junge Leute haben ihre »Clique « und finden sich bei den verschiedensten Gelegenheiten und an einschlägigen Orten zusammen. Alte Leute, die zu Hause alleine sind, suchen zum Beispiel einen Markt auf, um dort mit allen ihnen bekannten Händlern oder anderen Marktbesuchern zu schwätzen. Die Unterhaltung trägt zur Lebensqualität bei und ist dem alten Menschen mindestens so viel wert wie der Einkauf selbst, der eher Mittel zum sozialen Zweck bleibt (Gui 2005, 37). Der Einzelne begibt sich in Gesellschaft, um in Gesellschaft zu sein.

In Anbetracht dieses Bedürfnisses werden in der institutionellen Sozialen Arbeit Räume bereitgestellt und hergerichtet, die als »Produktionsstätte« für relationale Gü- ter dienen können. Ein Jugendtreff oder ein Altentreff wird vorgehalten, um bei »offener Tür « den Besuchern eine ihnen gefällige Konversation und Unterhaltung zu ermöglichen, wobei eine weitergehende Nutzung nicht ausgeschlossen ist. Auch Heime sollten dem Anspruch gerecht werden, nicht nur einzelne Zwecke stationärer Versorgung zu erfüllen, sondern den Bewohnern die Qualitäten eines geteilten Lebens wie Zugehörigkeit und Angenommensein zu verschaffen. Zudem ergibt sich in der gemeinsamen Bewältigung von Nöten, Leiden und Krisen die Erfahrung des Gutes personaler Teilhabe, das gerade in dieser Lage besonders geschätzt wird.

\section{Beziehungen beanspruchen Zeit}

Ob im informellen Miteinander oder in einer professionellen Arbeitsbeziehung, man braucht Zeit, um sich wirklich aufeinander einzulassen. Der Mangel an Zeit im Alltagsleben ist oft die Ursache für das Erfordernis einer außerhäuslichen Ver- geradezu Verarmung an sozial erbrachten Gütern bedeuten (Antoci/Sacco/Vanin 2008). Materielles Wohlergehen befriedigt ohne "Wohlstand an Beziehungen « (»relational wealth «) nicht nachhaltig. (Diwan 2000) In Sozialer Arbeit wie in der Pflege und in der Erziehung geht der Versuch, Effizienz durch Zeitersparnis zu erreichen, auf Kosten der Effektivität und ist nicht zielführend.

\section{Familie und \\ Lebensgemeinschaft als Gut}

Aus der ökonomischen Neueinschätzung des Wertes von Beziehungen ergibt sich eine Sicht auf die Familie (und analoge Lebensgemeinschaften) als einem Gut, das erarbeitet und gepflegt sein will.

Die Familie stellt Reziprozität zwischen den Generationen und den Geschlechtern her, verlangt sie auch und schafft ihnen in der familiären Gemeinschaft Wohlergehen. "The family is a srelational good inasmuch as it may only be generated and

\section{"Der Einzelne begibt sich in Gesellschaft, um in Gesellschaft $z u$ sein «}

sorgung: Kinder berufstätiger Eltern brauchen einen Platz in einer Tagesstätte, und häufig fehlt auch bei behinderten und pflegebedürftigen Angehörigen die Zeit, um sie in der Familie zu betreuen. Professionelle »Gefühlsarbeit « bietet aber keinen Ersatz, zumal dafür wiederum wenig Zeit vorgesehen ist ...

Generell wächst für uns alle im Fortschritt des Wirtschaftens der Zeitdruck. Um Zeit für die Arbeit, für Information und für den Konsum materieller Güter zu haben, spart man Zeit zum Leben ein. Den intensiv Beschäftigen droht Beziehungarmut. Sich auf Facebook zu »befreunden «, ersetzt nicht das Gut lebendiger Gemeinsamkeit.

Relationale Güter sind, weil sie viel Zeit und intensive Zuwendung beanspruchen, knapp. Ihr Wert steigt im Verhältnis zu warenförmigen Gütern, von denen immer mehr in kürzerer Zeit herstellen lassen. Wirtschaftliches Wachstum kann enjoyed together by those that compose it. It consists of relationships, not of individual goods - and not even in sum of individual goods. «(Donati 2007, 140) Von daher wird von Donati eine Familienpolitik gefordert, die der Beziehungsqualität in Familien zuträglich ist und sie stärkt.

Mit der Gewährung von Elternzeit ist die Politik einem unabweisbaren Bedarf nachgekommen. Die Entwicklung von Beziehungsqualität ist aber eine weit darüber hinausgehende Bildungsaufgabe. Eine Wertpräferenz für »Beziehungsglück « will behauptet, erläutert und vorgelebt werden. Ein relationales Gut ist keine konsumierbare Ware und nicht nach Lust und Laune verfügbar. Die normative Wertschätzung dieses Gutes fängt bei der Förderung von Freundschaft unter Kindern an und setzt sich in der Einübung von Partnerschaft fort, deren Gelingen sich gerade unter widrigen Umständen erweist. 
Die Erfahrung von Gemeinschaft in einem geteilten Leben bleibt nicht Sache des privaten Glücks; sie zu ermöglichen ist auch Aufgabe der Pflege sozialer Wohlfahrt.

\section{Literatur}

Antoci, Angelo/Sacco, Pier Luigi/Vanin, Paolo: Participation, Growth and Social Poverty: Social Capital in a Homogeneous Society. In: Open Economic Journal, 1/2008, S. 1-13.

Bruni, Luigino: Reciprocity, Altruism and the Civil Society. In praise of heterogeneity. Routledge, London 2008.

Diwan, Romesh: Relational Wealth and the Quality of Life. In: Journal of Socio-Economics, 29, 4, 2000. S. 305340.

Donati, Pierpaolo: Old and New Family Policies. The Perspectives of Relational Sociology. In: Sociologia, Problemas e Práticas, no. 54, 2007. S. 127 159.

Gui, Benedetto: On Relational Goods. Strategic implications of investment in relationships, In. International Journal of Social Economics, 23, 10/11, 1996. S. $260-278$.

Gui, Benedetto: From Transactions to Encounters: The Joint Generation of Relational Goods and Conventional Values. In: Gui, Benedetto / Sugden, Robert: Economics and Social Interaction. Accounting for Interpersonal Relations. Cambridge University Press, Cambridge 2005. S. 23-51.

Gui, Benedetto/Sugden, Robert: Economics and Social Interaction. Accounting for Interpersonal Relations. Cambridge University Press, Cambridge 2005.

Sacco, Pier Luigi/Vanin, Paolo/Zamagni, Stefano: The Economics of Human Relationships. In: Kolm, Serge-Christoph / Ythier, Jean Mercier: Handbook of the Economics of Giving, Altruism and Reciprocity. Volume I: Foundations. North Holland / Elsevier, Amsterdam 2006. S. 695-728.

Uhlaner, Carole Jean: >Relational Goods and Participation. Incorporating Sociability into a Theory of Rational Action. In: Public Choice, 62, 3, 1989. S. 253-285.

\section{Das neue Kompendium}

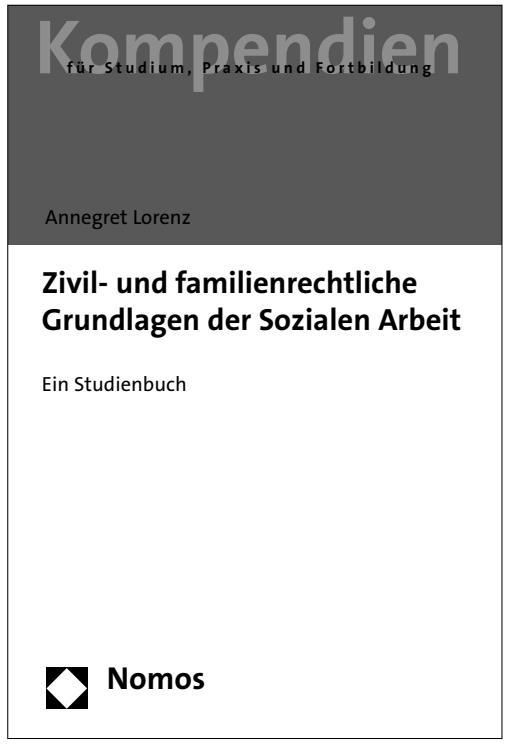

\section{Zivil- und familienrecht- liche Grundlagen der Sozialen Arbeit}

Von RAin Dr. Annegret Lorenz 2010, 332 S., brosch., 24,-€, ISBN 978-3-8329-5794-O

Erscheint ca. Oktober 2010
In den 1990er Jahren entwickelte sich die Soziale Arbeit aus den traditionellen Disziplinen Sozialpädagogik und Sozialarbeit. Dabei rückte das Recht stärker in den Fokus der Ausbildung, beschreibt es doch den Handlungsrahmen für die Soziale Arbeit. Zudem zählt seine Kenntnis in der Praxis zu den grundlegenden Beratungskompetenzen eines jeden Sozialarbeiters. Aufgrund der dynamischen Aktivität des Gesetzesgebers in diesem Bereich kommt es für Studierende nicht nur darauf an, die systemischen Grundlagen des Rechts zu beherrschen, sondern auch die Fähigkeit auszubilden, sich in die rechtlichen Neuerungen einzuarbeiten. Hier setzt das vorliegende Lehrbuch an.

Es vermittelt neben den für den Berufsalltag erforderlichen Grundlagen auch das methodisch juristische Rüstzeug zum professionellen Umgang mit neuen Rechtsmaterien. Die Darstellung beschränkt sich daher nicht auf die bloße Vermittlung von Rechtswissen. Vielmehr führen eine Vielzahl von Fallbeispielen und -lösungen den Studierenden an die eigenständige Arbeit mit dem Recht heran. Inhaltlich ist das vorliegende Studienbuch auf die zivilrechtlichen Grundlagen der Sozialen Arbeit beschränkt: In diesem Rahmen werden die bedeutsamen zivilrechtlichen Grundlagen behandelt. Daneben wird das Familienrecht - als Kernbereich der Ausbildung - eingehend dargestellt.

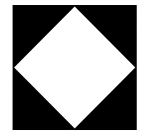

Nomos

Bitte bestellen Sie im Buchhandel oder

versandkostenfrei unter $\mathbf{w w w . n o m o s}$-shop.de 